\title{
A PSF-BASED APPROACH TO BIPLANE CALIBRATION IN 3D SUPER-RESOLUTION MICROSCOPY
}

\author{
Hagai Kirshner ${ }^{1, a}$, Thomas Pengo ${ }^{1, b},{\text { Nicolas } \text { Olivier }^{b}, \text { Daniel Sage }^{a} \text {, Suliana Manley }}^{b}$, Michael Unser $^{a}$ \\ ${ }^{a}$ Biomedical Imaging Group, EPFL, Lausanne, Switzerland \\ ${ }^{b}$ Laboratory for Experimental Biophysics, EPFL, Lausanne, Switzerland
}

\begin{abstract}
Super-resolution localization microscopy methods such as PALM and STORM have been shown to provide imaging with resolutions up to a few tens of nanometers while using relatively simple setups. Biplane PALM has extended the PALM technique to three-dimensions, by simultaneously using two imaging planes, with different focal depths. A key aspect in achieving good axial localization results is the alignment of the two planes. Currently available approaches assume that misaligned planes only result in scaling and rotation of the PSF pattern. We show in this work that this does not necessarily hold true, especially in the presence of refractive index mismatch between the different optical layers. Instead, we suggest a calibration algorithm that relies on a realistic PSF model and finds the affine transform that relates the two planes with respect to a point source in the object domain. Our calibration algorithm also determines the defocus distance between the planes.
\end{abstract}

Index Terms - super-resolution fluorescence microscopy, point spread function modeling, 3D particle localization.

\section{INTRODUCTION}

Recent advances in fluorescence microscopy have enabled overcoming the diffraction limit and achieving nanometer resolution, up to a tenfold increase with respect to confocal microscopy [1]. Localization microscopy techniques such as PALM or STORM achieve such an improvement in resolution by exciting and imaging each fluorescent molecule isolated from its neighbors, so that their position can be estimated with sub-pixel precision.

Extensions of the original concept have been proposed to also estimate the axial position of the molecules within the sample. These involve either shaping the PSF through a cylindrical lens [2], a phase mask [3], or simultaneously imaging at two different focal planes [4] (Biplane PALM). The latter has been demonstrated to achieve $30 \mathrm{~nm} \times 30 \mathrm{~nm}$ x $75 \mathrm{~nm}$ axial

This work was funded in part by the Euro BioImaging project, by the Indo Swiss Joint Research Programme, and by the Brazilian Swiss Joint Research Programme.

${ }^{1}$ These authors contributed equally to this work. resolution at depths of a few micrometers while using simple optical components [4]. Achieving such a resolution requires the two imaging planes to be well aligned. This can be done by a calibration procedure that extracts the $2 \mathrm{D}$ affine transformation (scaling, rotation, translation) between the two coordinate systems. The axial distance between the two imaging planes is an additional parameter that needs to be calibrated. The misalignment between the two planes was assumed in [4] to produce slight variations in the magnification and rotation between the images of the two planes. The scaling and rotation variations were then corrected by software and the axial distance was taken to be the design value. However, the axial distance between the planes gives rise to defocused PSF patterns which do not simply result in image scaling. Refractive index mismatch between the different layers introduce additional variations between the two PSF patterns. We also note that it is the particle location that the two planes need to calibrated for rather minimizing the differences between the two PSF patterns.

In this work we focus on the calibration procedure of the Biplane PALM microscope. Motivated by accuracy analysis of particle localization in super-resolution microscopy $[5,6$, $7,8]$, we use a realistic PSF model of a microscope that takes both defocusing and refractive index mismatch into account. We introduce a calibration procedure that uses a z-stack of fixed particles. Such a z-stack was already used in [4] for measuring the PSF of the microscope and we utilize it here in a different manner. In particular, we localize the fixed particles in the two planes and use this data to determine the affine transform between the coordinate systems of the two planes, as well as their axial distance. These parameters then can later be used by the Biplane PALM 3D localization algorithm.

\section{THE PSF MODEL}

We consider a microscopy setup where the biological sample is separated from the optical system by a coverslip and by a homogenous immersion medium. In nominal acquisition conditions, the point source fluorophore is located just beneath the coverslip and the detector plane is situated further away from the back focal plane so as to capture the in-focused Gaussian image of the fluorophore. The bi-plane setup con- 


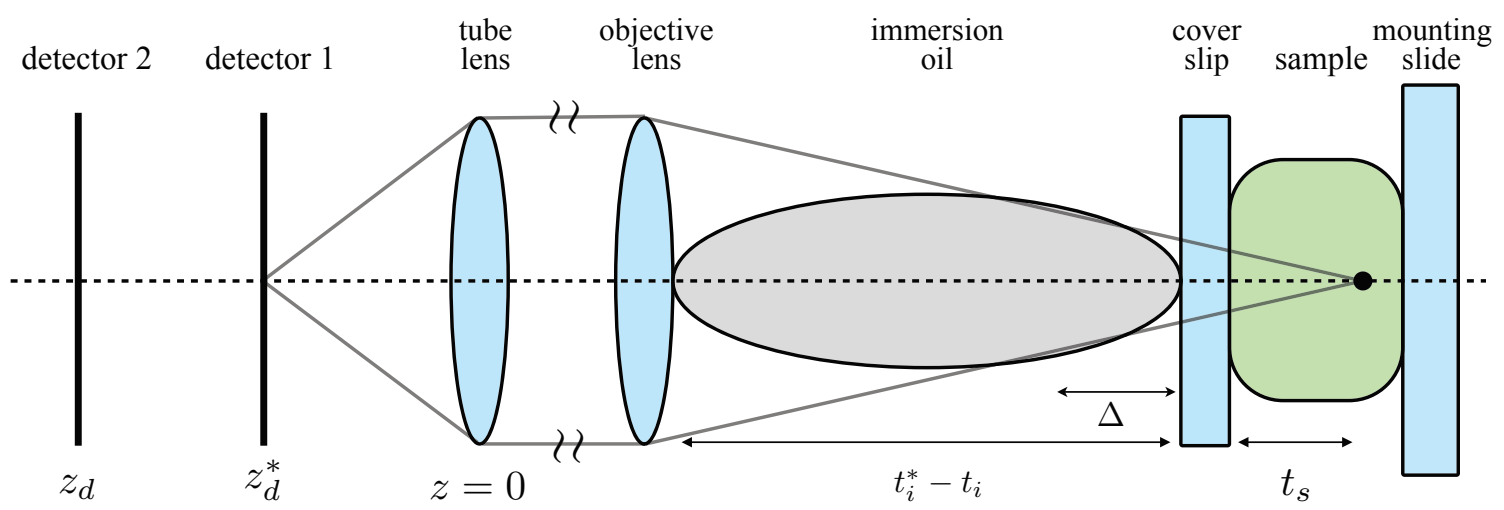

Fig. 1. A schematic of the biplane setup. A fluorophore that is located at a depth $t_{s}$ into the specimen is focused on the first detector plane by changing the thickness of the immersion layer $t_{i}^{*}-t_{i}$. The same fluorophore can be focused on the second detector plane by further changing the thickness by an amount of $\Delta$.

sists of a second detector plane that is located slightly further away; this plane captures a defocused image (Figure 1). We rely on the Gibson and Lanni PSF model, which accounts for the diffraction pattern in a microscope [9] in the presence of various aberrations. This model relies on Kirchhoff's diffraction formula at the back focal plane of the microscope. In nominal acquisition conditions the Gaussian image is located at the detector plane and the result is the Airy pattern. In such a case, there is no phase aberration in Kirchhoff's diffraction formula. When one of the nominal conditions is not met, however, the phase aberration at the back focal plane is no longer zero and it is given by $W(\rho)=k \cdot \operatorname{OPD}(\rho)$ where

$$
\begin{aligned}
& \mathrm{OPD}(\rho)=n_{s} t_{s}\left[1-\left(\frac{\mathrm{NA} \rho}{n_{s}}\right)^{2}\right]^{\frac{1}{2}}+ \\
& +n_{i}\left(t_{i}-t_{i}^{*}\right)\left[1-\left(\frac{\mathrm{NA} \rho}{n_{i}}\right)^{2}\right]^{\frac{1}{2}}+\frac{k a^{2}\left(z_{d}^{*}-z_{d}\right)}{2 z_{d}^{*} z_{d}} \rho^{2} .
\end{aligned}
$$

The last term of (1) corresponds to defocusing in the image plane. The parameters of (1) are described in Table 1 . We also note that a defocus measure of $z_{d}^{*}-z_{d}$ in image domain can be compensated for by moving the stage of the microscope by an amount of $\Delta=\frac{a^{2}\left(z_{d}^{*}-z_{d}\right) n_{i}}{z_{d}^{*} z_{d}}$. The PSF pattern is then given by

$h\left(x_{d}, y_{d}, z_{d}\right)=\frac{C}{z_{d}^{2}}\left|\int_{0}^{1} J_{0}\left[k a \rho \frac{\sqrt{x_{d}^{2}+y_{d}^{2}}}{z_{d}}\right] e^{i W(\rho)} \rho d \rho\right|^{2}$.

\section{THE ALGORITHM}

The calibration algorithm extracts the optical misalignment parameters between the two planes. Specifically, let $\mathbf{u}_{1}, \mathbf{u}_{2} \in$

\begin{tabular}{|c|c|}
\hline \multicolumn{2}{|c|}{ Name Description } \\
\hline$n_{s}$ & Refractive index of the specimen layer. \\
\hline$n_{i}$ & Refractive index of the immersion layer. \\
\hline$t_{i}, t_{i}^{*}$ & $\begin{array}{l}\text { Thickness of the immersion layer. An asterisk de- } \\
\text { notes nominal value. }\end{array}$ \\
\hline$t_{s}$ & Axial location of the fluorophore. \\
\hline$z_{d}, z_{d}^{*}$ & $\begin{array}{l}\text { Axial distance of the detector plane from the back } \\
\text { focal plane. } z_{d}^{*} \text { is approximately the tube length } \\
\text { value of the microscope. }\end{array}$ \\
\hline$x_{d}, y_{d}$ & Location at the detector plane. \\
\hline NA & Numerical aperture. \\
\hline$k$ & $\begin{array}{l}\text { Wave number of the light emitted by the fluo- } \\
\text { rophore. }\end{array}$ \\
\hline$a$ & $\begin{array}{l}\text { Aperture radius at the back focal plane: } a \cong \\
\mathrm{NA} z_{d}^{*} / M \text { where } M \text { is the magnification of the mi- } \\
\text { croscope. }\end{array}$ \\
\hline$\rho$ & Normalized radius of the aperture. \\
\hline
\end{tabular}

Table 1. Parameters of the Gibson and Lanni model

$\mathbb{R}^{2}$ describe the same location $(x, y)$ by means of two different coordinates systems, say detector 1 and detector 2 . The relation between these points is given by an affine transform $\mathbf{u}_{1}=\mathbf{A} \cdot \mathbf{u}_{2}+\mathbf{b}$ where $\mathbf{A}$ is a $2 \times 2$ matrix that describes scaling and rotation, and $\mathbf{b}$ is a $2 \times 1$ translation vector. The two detector planes are also located at different axial positions along the optical axis. Taking a practitioner point of view, the distance between the different locations $z_{d}-z_{d}^{*}$ can be expressed by means of stage displacement. The misalignment parameters are therefore $\mathbf{A}, \mathbf{b}$ and $\Delta$.

The input data to the algorithm is a z-stack of several fluorophores. The fluorophores are located at unknown 3D po- 

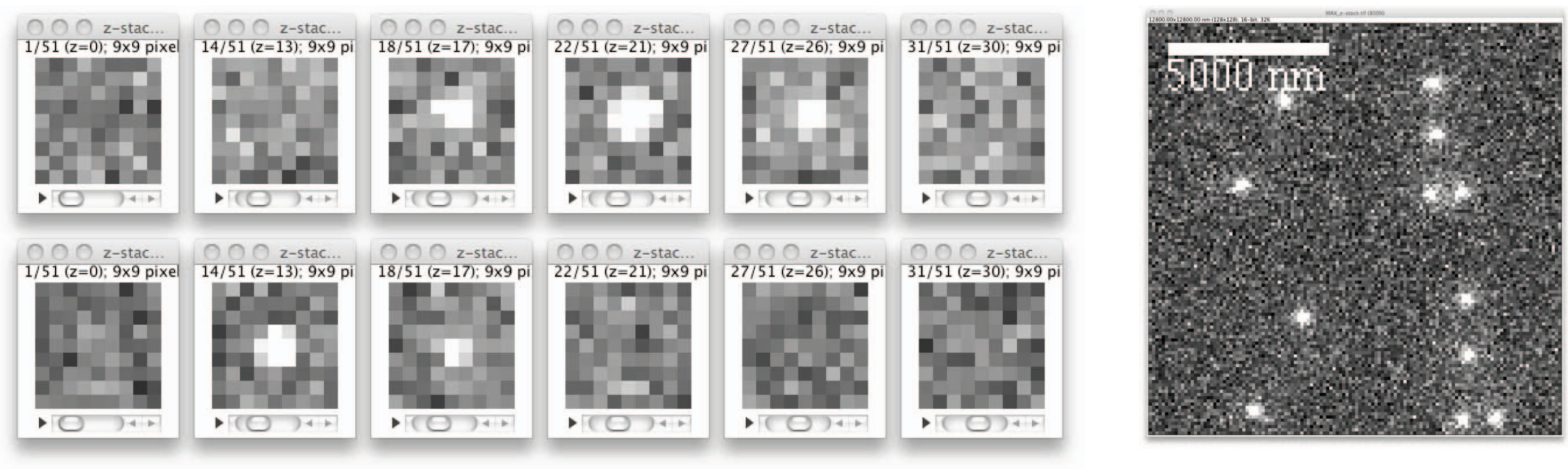

Fig. 2. An example of calibration z-stack data. Shown here on the left are slices of two z-stacks that include a single pointsource fluorophores. This fluorophore is captured by two planes which are 1,000[nm] apart along the optical axis in object domain. As the focal plane of the microscope moves away from the objective lens, the maximum response of the fluorophore appears at different slice indices. Our z-stack data simulator chooses random 3D positions for the fluorophores and computes the biplane acquisition images. Misalignment between the two planes is associated with 2D rotation, scaling and defocus. We also include Poisson-type photon emission and additive Gaussian noise models. Maximum projection of a typical calibration z-stack data is shown on the right.

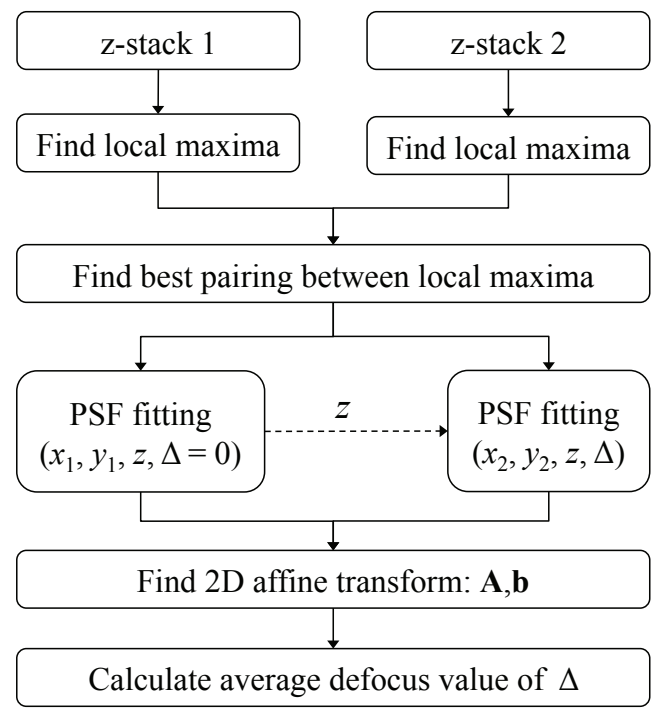

Fig. 3. A description of the calibration algorithm.

sitions in the specimen (Figure 2). Every slice in the stack is composed of two views - one for each detector plane. The main stages of the algorithm are illustrated in Figure 3. At first, the z-stack is split into two separate stacks - one for each detector plane. Every stack then undergoes local maxima identification. A local maxima is assumed to originate from a fluorophore; it is defined to be a voxel that is higher then its 26 neighbors and that meets a certain threshold value.
One of the advantages of using a theoretical PSF model, as we do here, is the ability to adapt the threshold to the stage displacement that represents every slice in the z-stack. The two sets of local maxima are then used for finding pairs of local maxima that minimize the following cost function

$$
\epsilon^{2}=\min _{\mathbf{A}, \mathbf{b}} \sum_{n}\left\|\mathbf{u}_{1, n}-\mathbf{A} \mathbf{u}_{2, n}-\mathbf{b}\right\|_{l_{2}}^{2}
$$

There are only few fluorophores and the algorithm checks all possible paring combination. For each combination it finds the best $\mathbf{A}, \mathbf{b}$ and calculates the error $\epsilon^{2}$. The pairing that corresponds to the smallest error is then chosen. The number of pairs is determined by the shortest local maxima list. The next stage consists of PSF fitting for every local maximum $\left(x_{n}, y_{n}, z_{n}\right)$ at the focused detector

$$
\begin{aligned}
\arg \min _{x_{d}, y_{d}, z_{d}} \sum_{x, y, z} \mid & h\left(x_{d}+x, y_{d}+y, z_{d}+z\right) \\
& -\left.I\left(x_{n}+x, y_{n}+y, z_{n}+z\right)\right|^{2},
\end{aligned}
$$

where $I$ is the given z-stack. We use the fast LevenbergMarquardt algorithm for fitting the pixel values with the PSF model that is parameterized by the axial and lateral positions of the fluorophore. The axial position that we find during the fitting process is assigned to its pair in the defocused detector and a second PSF fitting stage is carried out from this plane. The parameters that are found in this stage are the lateral position of the fluorophore and the defocus value $\Delta$. We compute the best affine transform that minimizes (3) while using the super-resolved locations of each particle. The output of the algorithms are the three misalignment parameters: A,b and the average value of $\Delta$ among all pairs. Finding the best affine transform corresponds to solving a set of linear equations. 


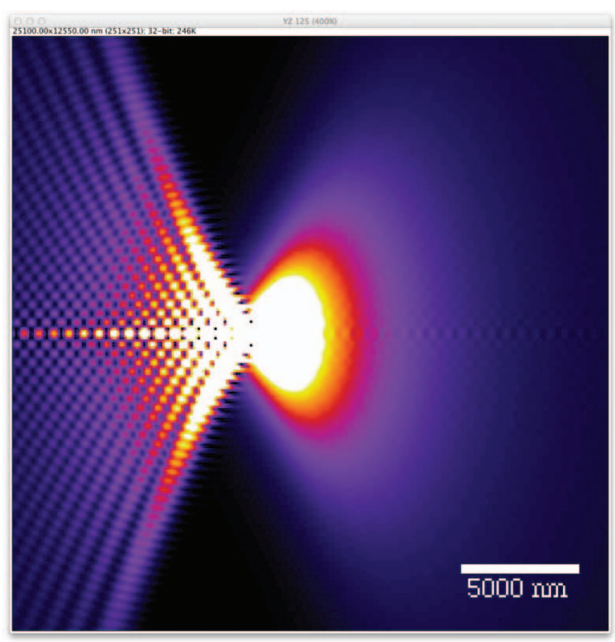

Fig. 4. $y z$ section of a Gibson and Lanni PSF pattern for a particle depth of 2,000 [nm]. The simulation parameters are $\mathrm{NA}=1.4, n_{i}=1.5, n_{s}=1.33, \lambda=500[\mathrm{~nm}]$, the pixel size is $50[\mathrm{~nm}]$ and the axial resolution is 100 [nm]. The PSF pattern is non-symmetric due to the refractive index mismatch.

\section{RESULTS}

We implemented the proposed algorithm in ImageJ and tested it on simulated data. The PSF model allows one to introduce refractive index mismatch, which results in a non-symmetric PSF pattern (Figure 4). This, in turn, allows us to achieve a relatively high localization accuracy [8]. Calibration results are given in Table 2, demonstrating the accuracy of the proposed approach. The Table shows results based on six particles that are randomly located in a $3[\mu \mathrm{m}]$ specimen. The z-stack is of size $128 \times 128 \times 50$. The acquisition parameters were $\mathrm{NA}=1.4, M=100, n_{i}=1.5, n_{s}=1.33$, $\lambda=500[\mathrm{~nm}]$. The pixel size was $100[\mathrm{~nm}]$ and so was the axial resolution value. Every set of parameters was realized 10 times for which the average value was taken. Every realization included with an additive Gaussian noise with $\sigma=$ 2,500 ; the maximum value in the $\mathrm{z}$-stack was 65,535 .

\section{CONCLUSIONS}

We introduced a model-based approach for biplane microscopy calibration. We showed that in the presence of refractive index mismatch, the PSF patterns of the two imaging planes do not differ by scaling alone, making currently available calibration methods difficult to use. Our algorithm, on the other hand, uses a 3D PSF model that accounts for refractive index mismatch and for defocusing. The calibration data consists of a z-stack of fixed fluorophores which is already being used as part of the current calibration procedure. Localization results were shown to achieve precision of the
Table 2. Monte Carlo calibration experiments

\begin{tabular}{|c|c|c|}
\hline \multirow{2}{*}{ Parameters $^{1}$} & \multicolumn{2}{|c|}{ Estimated values } \\
\cline { 2 - 3 } & MSE $^{2}$ & $\Delta$ \\
\hline$(1.0,110,500)$ & 0.017 & 500 \\
$(0.9,110,500)$ & 0.015 & 503 \\
$(1.1,110,500)$ & 0.589 & 508 \\
$(1.1,80,990)$ & 0.016 & 991 \\
$(0.9,80,990)$ & 0.017 & 1003 \\
$(1.0,80,990)$ & 0.016 & 985 \\
\hline 1
\end{tabular}

order of a few nanometers radially and a few tens of nanometers axially. The calibration parameters were estimated to a good precision, too.

\section{REFERENCES}

[1] G. Patterson, M. Davidson, S. Manley, and J. LippincottSchwartz, "Superresolution imaging using single-molecule localization," Апnи Rev Phys Chem., vol. 61, pp. 345-67, March 2010.

[2] B. Huang, W. Wang, M. Bates, and X. Zhuang, "Threedimensional super-resolution imaging by stochastic optical reconstruction microscopy," Science, vol. 319, no. 5864, pp. 810 813, February 2008.

[3] S. Pavani, M. Thompson, J. Biteen, S. Lord, N. Liu, R. Twieg, R. Piestun, and W. Moerner, "Three-dimensional, singlemolecule fluorescence imaging beyond the diffraction limit by using a double-helix point spread function," PNAS, vol. 106, no. 9, pp. 2995-2999, March 2009.

[4] M. Juette, T. Gould, M. Lessard, M. Mlodzianoski, B. Nagpure, B. Bennet, S. Hess, and J. Bewersdorf, "Three-dimensional sub$100 \mathrm{~nm}$ resolution fluorescence microscopy of thick samples," Nature Methods, vol. 5, no. 6, pp. 527-529, June 2008.

[5] R. J. Ober, S. Ram, and E. S. Ward, "Localization accuracy in single-molecule microscopy," Bio- physical Journal, vol. 86, no. 2, pp. 1185-1200, February 2004.

[6] F. Aguet, D. Van De Ville, and M. Unser, "A maximumlikelihood formalism for sub-resolution axial localization of fluorescent nanoparticles," Optics Express, vol. 13, no. 26, pp. 10503-10522, December 2005.

[7] B. Zhang, J. Zerubia, and J.-C. Olivo-Marin, "Gaussian approximations of fluorescence microscope point-spread function models," Applied Optics, vol. 46, no. 10, pp. 1819-1829, April 2007.

[8] S. Ram, J. Chao, P. Prabhat, E.S. Ward, and R.J. Ober, "A novel approach to determining the three-dimensional location of microscopic objects with applications to $2 \mathrm{~d}$ particle tracking," Proc. SPIE, vol. 6443, pp. 64430D-1-64430D-7, 2007.

[9] S. Frisken-Gibson and F. Lanni, "Experimental test of an analytical model of aberration in an oil-immersion objective lens used in three-dimensional light microscopy," J. Opt. Soc. Am. A, vol. 9, no. 1, pp. 154-166, January 1992, originally published in J. Opt. Soc. Am. A 8, 1601-1613 (1991). 